\title{
Daunorubicin Hydrochloride
}

National Cancer Institute

\section{Source}

National Cancer Institute. Daunorubicin Hydrochloride. NCI Thesaurus. Code C1583.

The hydrochloride salt of an anthracycline antineoplastic antibiotic with therapeutic effects similar to those of doxorubicin. Daunorubicin exhibits cytotoxic activity through topoisomerase-mediated interaction with DNA, thereby inhibiting DNA replication and repair and RNA and protein synthesis. 